\title{
Are all DMARDs equivalent?
}

\author{
Peter E Lipsky
}

A comprehensive review by Donahue et al. of rheumatoid arthritis (RA) therapy using diseasemodifying antirheumatic drugs (DMARDs) concludes that differences in efficacy among these agents are difficult to discern (Donahue KE et al. [2008] Ann Intern Med 148: 124-134). This finding is not unexpected, considering the general nature of the studies assessed, but it could have substantial adverse implications for the care of patients with RA. The next issue of Nature Clinical Practice Rheumatology will present an in-depth analysis of this review by leading experts in the field.

The results of Donahue and colleagues' review are not dissimilar to those of a similar, earlier review (Felson DT et al. [1990] Arthritis Rheum 33: 1449-1461) of the then-available DMARDS-gold salts, D-penicillamine, sulfasalazine, and methotrexate-in that all of the agents studied showed broadly equivalent effects. Time and experience have, however, indicated that these agents are not interchangeable; gold salts and D-penicillamine are rarely, if ever, used today - most patients are treated with methotrexate, with or without glucocorticoids. Donahue and colleagues' review also includes newer chemical DMARDs and agents that neutralize tumor necrosis factor, but their conclusions are similar to those of Felson et al.

What could be responsible for the apparent lack of differences in efficacy between DMARDs? First, most of the studies included in the review were carried out by pharmaceutical companies for the purpose of licensing a product or expanding its indicated uses. As these short-term trials include highly selected patient populations, they often fail to reflect 'real life' clinical practice and, consequently, might not demonstrate the full spectrum of variables that determine whether or not an agent is effective. Moreover, the trials were
[These trials] ... might not demonstrate the full spectrum of variables that determine whether or not an agent is effective

PE Lipsky is the Editor-inChief of Nature Clinical Practice Rheumatology.

\section{Competing interests}

The author declared no competing interests.

www.nature.com/clinicalpractice doi: $10.1038 /$ ncprheum0800 often carried out at a time or place in which the medication might have been perceived to be the only available therapeutic option, which could have artificially bolstered patients' compliance. Furthermore, many of the outcome measures used in clinical trials of RA might lack a sufficiently large linear range to distinguish differences in effect size between therapeutic agents with accuracy. Finally, timing of the introduction of therapeutic agents and the use of objective measures to determine when to alter the treatment regimen might have had an important effect on overall outcome, as has been demonstrated (Goekoop-Ruiterman YP et al. [2005] Arthritis Rheum 52: 3381-3390).

In view of these considerations, the results of the review by Donahue et al. are not unexpected; their possible implications are, however, of concern. Biologic DMARDs are very expensive and, therefore, not widely available or are restricted in their use. We can only hope that the results of the review are not used to restrict use of biologic DMARDs further because, as concluded by Donahue et al., "Limited available comparable evidence does not support one monotherapy over another for adults with rheumatoid arthritis". Data indicating that strategies employing biologic DMARDs can be more effective than other approaches are clearly emerging (Goekoop-Ruiterman YP et al. [2005] Arthritis Rheum 52: 3381-3390). The rheumatology community must perform the appropriate trials to determine whether the most frequently used chemical and biologic DMARDs and current strategies are indeed the most effective for treating patients with RA. A great disservice would be done to individuals with RA if their therapeutic options were restrained by acceptance of the conclusions of a review of trials that were not really designed to address issues of comparative effectiveness in clinical practice. 